\title{
An overlapping additive Schwarz preconditioner for boundary element approximations to the Laplace screen and Lamé crack problems
}

\author{
T. TRAN ${ }^{*}$ and E.P.STEPHAN ${ }^{\dagger}$
}

Dedicated to Professor Erwin Stein on the occasion of his $70^{\text {th }}$ birthday.

Received March 9, 2003

Received in revised form July 22, 2004

\begin{abstract}
We study a two-level overlapping additive Schwarz preconditioner for the $h$-version of the Galerkin boundary element method when used to solve hypersingular integral equations of the first kind on an open surface in $\mathbb{R}^{3}$. These integral equations result from Neumann problems for the Laplace and Lamé equations in the exterior of the surface. We prove that the condition number of the preconditioned system is bounded by $O\left(1+\log ^{2}(H / \delta)\right)$, where $H$ denotes the diameter of the subdomains and $\delta$ the size of the overlap.
\end{abstract}

Keywords: Galerkin boundary element method, $h$ version, additive Schwarz, overlapping, preconditioned conjugate gradient

\section{INTRODUCTION}

In this paper we consider an overlapping additive Schwarz method for the Galerkin boundary element approximation of the Laplace screen and Lamé crack problems in three dimensions with Neumann boundary conditions. In the context of boundary element methods, these problems are reformulated into hypersingular integral equations on the screen, the solutions of which are then approximated with spline functions by the $h$-version of the Galerkin method (see details in Section 2).

It is well known that the linear algebraic systems arising from these approximations can be efficiently solved with preconditioning techniques using domain decompositions. Substructuring techniques have been studied $[1,2,8]$ for hypersingular integral equations on surfaces in $\mathbb{R}^{3}$. For equations on curves in $\mathbb{R}^{2}$, overlapping additive Schwarz methods have been discussed in $[15,17]$ for both the $h$ - and $p$ -

\footnotetext{
${ }^{*}$ School of Mathematics, University of New South Wales, Sydney 2052, Australia

† Institut für Angewandte Mathematik, University of Hannover, 30167 Hannover, Germany

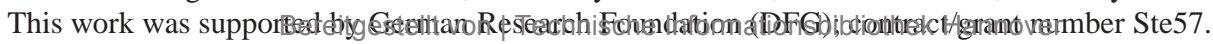


versions. For the case of surfaces in $\mathbb{R}^{3}$ where the $p$-version is used on anisotropic elements, this type of preconditioners has been studied in [9]. However, they have not been thoroughly addressed in case the $h$-version is used for these integral equations on surfaces. The purpose of the work in this paper is to fill this gap.

We prove that the condition number of the preconditioned system is bounded by $O\left(1+\log ^{2}(H / \delta)\right)$, where $H$ is the diameter of the subdomains, and $\delta$ is the size of the overlaps. We note that the overlapping subdomains are not required to be quadrilaterals, in fact their boundaries can be zigzag closed lines (see Fig. 1).

In the finite element literature, overlapping additive Schwarz methods have been discussed in e.g. [6,12]. The algorithm in [12] consists of mainly two steps. The first step involves the elimination of interior unknowns by using Schur complements. The second step involves subspace decomposition with subspaces being vertex spaces. The algorithm is different from the one analysed in the present paper.

In [6] there is a very brief sketch of the proof in the 3-dimensional case of differential operators, which corresponds to our situation of hypersingular integral operators on a surface in $\mathbb{R}^{3}$ with the $H_{00}^{1 / 2}$ or $\widetilde{H}^{1 / 2}$ norm. A detailed analysis of the situation is not given there. We believe that our analysis, going over several pages, is by no means trivial.

The paper is organised as follows. In Section 2 we introduce the model problems and the resulting hypersingular integral equations, together with their Galerkin approximations. The abstract framework of the additive Schwarz method is presented in Section 3. In Section 4 we define the overlapping subdomains and the resulting subspace decomposition, and state the main result of the paper. The proof is carried out by proving different lemmas in Section 5. Numerical experiments for both the Laplace and Lamé operators are presented in Section 6.

\section{MODEL PROBLEMS AND BOUNDARY-INTEGRAL EQUATIONS}

Let $\Gamma \subset \mathbb{R}^{3}$ be an open two dimensional surface with a polygonal boundary, and let $\mathbf{n}$ be a normal vector to $\Gamma$, which defines two sides $\Gamma_{1}$ and $\Gamma_{2}$ of $\Gamma$. In the unbounded domain $\Omega=\mathbb{R}^{3} \backslash \bar{\Gamma}$, we consider the following Neumann problem, deferring the definitions of the usual Sobolev spaces to a later paragraph in the section.

Given two functions $f_{1}, f_{2} \in\left(H^{-1 / 2}(\Gamma)\right)^{d}, d=1$ or $d=3$, such that $f:=f_{1}-f_{2}$ $\in\left(\widetilde{H}^{-1 / 2}(\Gamma)\right)^{d}$, find $U \in\left(H_{\text {loc }}^{1}(\Omega)\right)^{d}$ satisfying

$$
\begin{array}{ll}
\mathscr{L} U=0 & \text { in } \Omega \\
\mathscr{B}_{i} U=f_{i} & \text { on } \Gamma_{i}, \quad i=1,2
\end{array}
$$

where

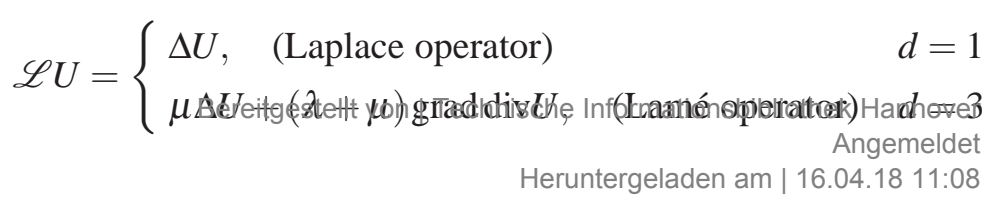


and

$$
\mathscr{B}_{i} U= \begin{cases}\left.\frac{\partial U}{\partial \mathbf{n}}\right|_{\Gamma_{i}}, & d=1 \\ \left.T U\right|_{\Gamma_{i}}, & d=3\end{cases}
$$

with $T U \in \mathbb{R}^{3}$ being the traction defined by

$$
T U=\lambda(\operatorname{div} U) \mathbf{n}+\mu \frac{\partial U}{\partial \mathbf{n}}+\mu \mathbf{n} \times \operatorname{curl} U .
$$

Here $\lambda$ and $\mu$ are the Lamé constants satisfying $\mu>0$ and $2 \lambda+\mu>0$. Additionally, the vanishing condition $U(x)=O(1 /|x|)$ is required at infinity.

The problem (2.1) can be reformulated into hypersingular integral equations of the first kind on the two dimensional open surface $\Gamma$. In order to do so, we first note that the fundamental solution of the differential operator $\mathscr{L}$ is given by

$$
G(x, y)= \begin{cases}\frac{1}{2 \pi} \frac{1}{|x-y|}, & d=1 \\ \frac{\lambda+3 \mu}{4 \pi \mu(\lambda+2 \mu)}\left\{\frac{1}{|x-y|} I_{3 \times 3}+\frac{\lambda+\mu}{\lambda+3 \mu} \frac{(x-y)(x-y)^{t}}{|x-y|^{3}}\right\}, & d=3\end{cases}
$$

where $x, y \in \mathbb{R}^{3}, I_{3 \times 3}$ is the identity matrix of size $3 \times 3$, and $t$ denotes the transpose of the matrix. Let $u=\left.[U]\right|_{\Gamma}$ be the jump of $U$ across $\Gamma$. Then the problem (2.1) is equivalent to (see $[13,14])$

$$
D u=g
$$

where $D:\left(\widetilde{H}^{1 / 2}(\Gamma)\right)^{d} \rightarrow\left(H^{-1 / 2}(\Gamma)\right)^{d}$ is the hypersingular integral operator defined as

$$
D u(x):= \begin{cases}-\frac{\partial}{\partial \mathbf{n}_{x}} \int_{\Gamma} \frac{\partial}{\partial \mathbf{n}_{y}} G(x, y) u(y) \mathrm{d} s_{y}, & d=1 \\ -T_{x} \int_{\Gamma}\left(T_{y} G(x, y)\right)^{t} u(y) \mathrm{d} s_{y}, & d=3\end{cases}
$$

and

$$
g(x):= \begin{cases}f_{1}(x)+f_{2}(x)-\int_{\Gamma}\left(f_{1}(x)-f_{2}(x)\right) \frac{\partial}{\partial \mathbf{n}_{y}} G(x, y) \mathrm{d} s_{y}, & d=1 \\ f_{1}(x)+f_{2}(x)-\int_{\Gamma}\left(f_{1}(x)-f_{2}(x)\right) T_{y} G(x, y) \mathrm{d} s_{y}, & d=3 .\end{cases}
$$

In order to describe the operator properties of $D$, we now give the definitions for the function spaces mentioned above. Let $S \subset \mathbb{R}^{n}, n=1$ or 2, be a Lipschitz domain. We denote by $L_{2}(S)$ the usual space of Lebesgue square integrable functions equipped with the standard norm $\|\cdot\|_{L_{2}(S)}$, and by $H^{1}(S)$ the usual Sobolev space equipped with the norm 
where $|v|_{H^{1}(S)}=\|\nabla v\|_{L_{2}(S)}$. The space $H^{1 / 2}(S)$ is defined by Hilbert space interpolation [3] so that

$$
H^{1 / 2}(S):=\left[L_{2}(S), H^{1}(S)\right]_{1 / 2}
$$

with the norm

$$
\|u\|_{H^{1 / 2}(S)}^{2}=\int_{0}^{\infty} K(t, u)^{2} \frac{\mathrm{d} t}{t^{2}}
$$

where the $K$-functional is defined, for $u \in L_{2}(S)+H^{1}(S)$, by

$$
K(t, u)^{2}=\inf _{u=u_{0}+u_{1}}\left(\left\|u_{0}\right\|_{L_{2}(S)}^{2}+t^{2}\left\|u_{1}\right\|_{H^{1}(S)}^{2}\right) .
$$

Similarly, we define the subspace $\widetilde{H}^{1 / 2}(S)$ by

$$
\widetilde{H}^{1 / 2}(S):=\left[L_{2}(S), H_{0}^{1}(S)\right]_{1 / 2}
$$

with the norm

$$
\|u\|_{\widetilde{H}^{1 / 2}(S)}^{2}=\int_{0}^{\infty} K(t, u)^{2} \frac{\mathrm{d} t}{t^{2}}
$$

where the $K$-functional is defined, for $u \in L_{2}(S)+H_{0}^{1}(S)$, by

$$
K(t, u)^{2}=\inf _{u=u_{0}+u_{1}}\left(\left\|u_{0}\right\|_{L_{2}(S)}^{2}+t^{2}\left\|u_{1}\right\|_{H^{1}(S)}^{2}\right) .
$$

The spaces $H^{-1 / 2}(S)$ and $\widetilde{H}^{-1 / 2}(S)$ are defined as the dual spaces of $\widetilde{H}^{1 / 2}(S)$ and $H^{1 / 2}(S)$, respectively, with respect to the usual extension of the $L_{2}$ inner product on $S$.

The spaces $\left(\widetilde{H}^{1 / 2}(S)\right)^{d}$ and $\left(H^{1 / 2}(S)\right)^{d}$ for $d=3$ are defined as spaces of functions in $\widetilde{H}^{1 / 2}(S)$ and $H^{1 / 2}(S)$ componentwise.

It was shown in $[13,14]$ that the operator $D$ defined in (2.3) is a bijective mapping from $\left(\widetilde{H}^{1 / 2}(\Gamma)\right)^{d}$ onto $\left(H^{-1 / 2}(\Gamma)\right)^{d}$. Moreover (see also [7]), if

$$
a(v, w):=\langle D v, w\rangle \quad \forall v, w \in\left(\widetilde{H}^{1 / 2}(\Gamma)\right)^{d}
$$

(where $\langle D v, w\rangle$ denotes the duality pairing which coincides with the $L_{2}$ inner product on $\Gamma$ if $\left.D v, w \in\left(L_{2}(\Gamma)\right)^{d}\right)$ then $a(\cdot, \cdot)$ is a positive-definite and symmetric bilinear form on $\left(\widetilde{H}^{1 / 2}(\Gamma)\right)^{d}$ satisfying

$$
a(v, v) \simeq\|v\|_{\left(\widetilde{H}^{1 / 2}(\Gamma)\right)^{d}}^{2} \quad \forall v \in\left(\widetilde{H}^{1 / 2}(\Gamma)\right)^{d} .
$$

Here, and in what follows, the notation $a \preceq b$ is used to indicate that $a \leqslant c b$ for some positive constant $c$ that is independent of the main quantities of interest, while $a \simeq b$ is equivalent to $a \preceq b$ and $b \preceq a$.

A weak form of equation (2.2) is the problem of finding

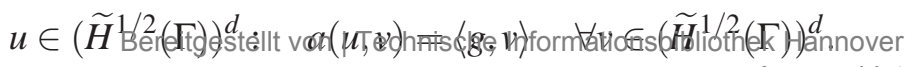


The problem (2.5) will be approximated by first constructing a finite-dimensional subspace $\mathscr{S} \subset\left(\widetilde{H}^{1 / 2}(\Gamma)\right)^{d}$, and then finding

$$
u_{\mathscr{S}} \in \mathscr{S}: \quad a\left(u_{\mathscr{S}}, v\right)=\langle g, v\rangle \quad \forall v \in \mathscr{S} .
$$

In the remainder, for notational convenience we will consider only the case $d=1$. The case $d=3$ should be treated similarly.

\section{ABSTRACT FRAMEWORK OF ADDITIVE SCHWARZ METHODS}

Additive Schwarz methods provide fast solutions to equation (2.6) by solving (at the same time) problems of smaller size. Let $\mathscr{S}$ be decomposed as

$$
\mathscr{S}=\mathscr{S}_{0}^{\prime}+\cdots+\mathscr{S}_{J}^{\prime}
$$

where $\mathscr{S}_{i}^{\prime}, i=0, \ldots, J$, are subspaces of $\mathscr{S}$. Let $b_{i}(\cdot, \cdot): \mathscr{S}_{i}^{\prime} \times \mathscr{S}_{i}^{\prime} \rightarrow \mathbb{R}$ be a bilinear form defined on $\mathscr{S}_{i}^{\prime}$ and $P_{i}: \mathscr{S} \rightarrow \mathscr{S}_{i}^{\prime}$ be a projection defined by

$$
b_{i}\left(P_{i} v, w\right)=a(v, w) \quad \forall v \in \mathscr{S}, \quad \forall w \in \mathscr{S}_{i}^{\prime} .
$$

Then the additive Schwarz method for equation (2.6) consists in solving, by an iterative method, the equation

$$
P u_{\mathscr{S}}=g
$$

where $P:=P_{0}+\cdots+P_{J}$ is the additive Schwarzoperator and $g$ is given by $g=\sum_{i=0}^{J} g_{i}$, with $g_{i} \in \mathscr{S}_{i}^{\prime}$ being solutions of

$$
b_{i}\left(g_{i}, w\right)=\langle g, w\rangle \quad \forall w \in \mathscr{S}_{i}^{\prime} .
$$

The equivalence of (2.6) and (3.2) was discussed in [16]. The operator $P$ can be considered as the preconditioned version of $D$, i.e., $P=B D$ with some preconditioner $B$. In the implementation of the method, e.g., with the conjugate gradient method, one is interested in computing $B^{-1} r$ for a residual $r \in \mathscr{S}$ :

\section{Additive Schwarz Algorithm:}

1. Subspace corrections: For $j=0, \ldots, J$, find $u_{j} \in \mathscr{S}_{j}^{\prime}$ such that

$$
b_{j}\left(u_{j}, v_{j}\right)=\left\langle r, v_{j}\right\rangle \quad \forall v_{j} \in \mathscr{S}_{j}^{\prime} .
$$

2. Preconditioned residual:

$$
B^{-1} r=\sum_{j=0}^{J} u_{j} .
$$

Bounds for the minimum and maximum eigenvalues of the additive Schwarz operator $P$ can be obtained byusing the following lemma (see [1 0.181) 
Lemma 3.1. (i) If there exists a positive constant $C_{0}$ such that for any $u \in \mathscr{S}$ and $u_{i} \in \mathscr{S}_{i}^{\prime}$ satisfying $u=\sum_{i=0}^{J} u_{i}$ there holds

$$
a(u, u) \leqslant C_{0} \sum_{i=0}^{J} b_{i}\left(u_{i}, u_{i}\right)
$$

then

$$
\lambda_{\max }(P) \leqslant C_{0} .
$$

(ii) If there exists a positive constant $C_{1}$ such that any $u \in \mathscr{S}$ has a decomposition $u=\sum_{i=0}^{J} u_{i}$ satisfying

$$
\sum_{i=0}^{J} b_{i}\left(u_{i}, u_{i}\right) \leqslant C_{1} a(u, u)
$$

then

$$
\lambda_{\min }(P) \geqslant C_{1}^{-1} .
$$

\section{OVERLAPPING ADDITIVE SCHWARZ PRECONDITIONER}

\subsection{Boundary element subspace}

In this subsection we define the finite-dimensional subspace $\mathscr{S}$ in (2.6) on a twolevel grid.

The coarse grid: Assume that $\Gamma$ is partitioned into subdomains $\Gamma_{i}, i=1, \ldots, J$, where each subdomain $\Gamma_{i}$ is the image of the reference square $\hat{R}=(-1,1)^{2}$ under a smooth bijective mapping $\mathscr{F}_{i}: \hat{R} \rightarrow \Gamma_{i}$. Denoting by $H$ the diameter of the subdomains, we assume that

$$
\left\|J_{\mathscr{F}_{i}}\right\|_{L_{\infty}(\hat{R})} \preceq H, \quad\left\|J_{\mathscr{F}_{i}^{-1}}\right\|_{L_{\infty}(\hat{R})} \preceq H^{-1}
$$

where $J_{\mathscr{F}_{i}}$ denotes the Jacobian matrix of the transformation and the norm is a matrix norm. The partition is assumed to be conforming in the sense that the nonempty intersection of a pair of distinct subdomains is a single common vertex or edge of both subdomains, and that each vertex of the domain $\Gamma$ coincides with at least one subdomain vertex.

We define on this coarse grid the space $\mathscr{S}$ of continuous piecewise bilinear functions, vanishing on the boundary of $\Gamma$.

The fine grid: Each subdomain $\Gamma_{i}$ is further divided into disjoint quadrilateral or triangular elements, giving a locally uniform mesh of element of size $h_{i}$ in $\Gamma_{i}$. We denote by $h$ the maximum value of $h_{i}, i=1, \ldots, J$.

The finite-dimensional space $\mathscr{S}$ is defined as the space of continuous piecewisebilinear functions (in the case of quadrilateral elements) or piecewise-linear functions (in the case of triangular elements) on the fine grid, vanishing on the boundary of $\Gamma$. 

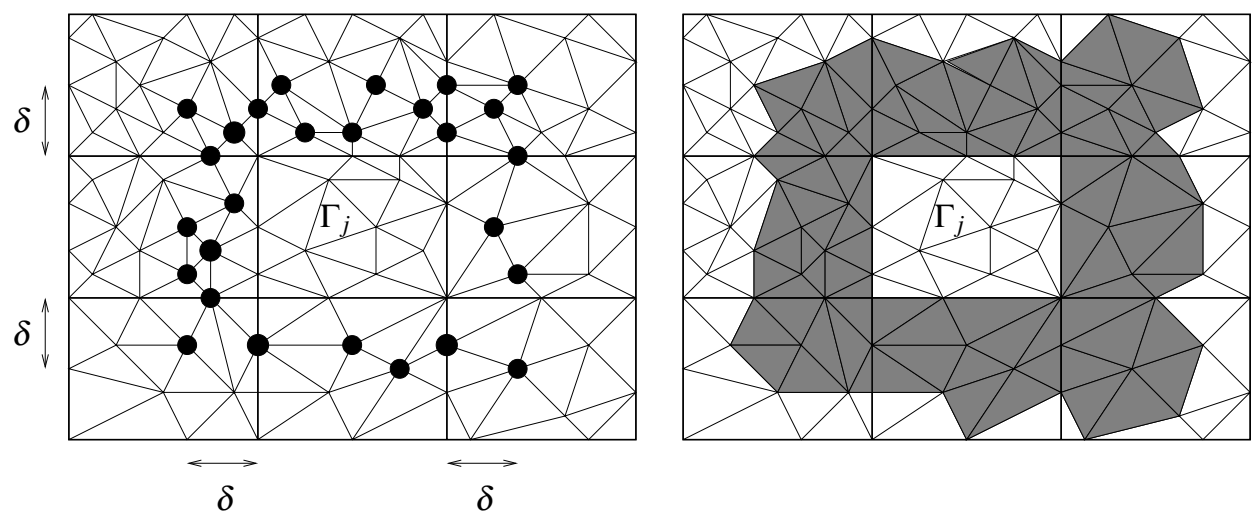

Figure 1. - vertex at a distance $\delta$ to $\bar{\Gamma}_{j}, \tilde{\Gamma}_{j}$ : shaded region, $\Gamma_{j}^{\prime}=\Gamma_{j} \cup \tilde{\Gamma}_{j}$ : overlapping subdomain, $R_{j}$ : (rectangle in shaded region) support of cutoff function $\vartheta_{j}$.

We denote by $\left\{\mathbf{x}_{k}: k \in \mathscr{N}\right\}$ the set of all vertices of elements in the fine grid which are not on the boundary of $\Gamma$, and by $\left\{\varphi_{k}: k \in \mathscr{N}\right\}$ the set of nodal basis functions in $\mathscr{S}$, i.e., $\varphi_{k}\left(\mathbf{x}_{l}\right)=\delta_{k l}$. We also define subspaces $\mathscr{S}_{j}=\mathscr{S} \cap \widetilde{H}^{1 / 2}\left(\Gamma_{j}\right)$ of functions in $\mathscr{S}$ supported in $\bar{\Gamma}_{j}$.

\subsection{Overlapping subdomains}

We extend each subdomain $\Gamma_{j}$ in the following way. First we define, for some $\delta>0$ called the overlap size,

$$
\tilde{\mathscr{S}}_{j}=\operatorname{span}\left\{\varphi_{k}: \mathbf{x}_{k} \notin \bar{\Gamma}_{j}, \operatorname{dist}\left(\mathbf{x}_{k}, \partial \Gamma_{j}\right) \leqslant \delta\right\}
$$

and denote

$$
\tilde{\Gamma}_{j}=\operatorname{supp}\left\{\varphi_{k}: \varphi_{k} \in \tilde{\mathscr{S}}_{j}\right\}
$$

which is the shaded area in Fig. 1. (Here the distance is defined with the max norm $\|\mathbf{x}\|=\max \left\{\left|x_{1}\right|,\left|x_{2}\right|\right\}$ where $\mathbf{x}=\left(x_{1}, x_{2}\right)$.) The extended subdomain $\Gamma_{j}^{\prime}$ is then defined as $\Gamma_{j}^{\prime}=\bar{\Gamma}_{j} \cup \tilde{\Gamma}_{j}$. We note that $\Gamma_{j}^{\prime}$ need not be a quadrilateral domain. Also, if $\delta$ is chosen such that $\delta \in(0, H]$, then

$$
\operatorname{diam}\left(\Gamma_{i}^{\prime}\right) \simeq H
$$

\subsection{Subspace decomposition}

The decomposition (3.1) is performed with subspaces $\mathscr{S}_{j}, j=0,1, \ldots, J$, defined as

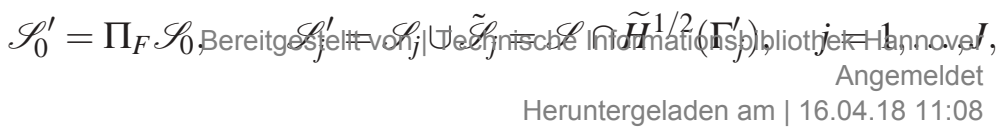


where $\Pi_{F}$ is the interpolation operator which interpolates continuous functions into functions in $\mathscr{S}$.

The bilinear forms $b_{i}(\cdot, \cdot)$ associated with the subspaces $\mathscr{S}_{i}^{\prime}$ (see Section 3) are defined as

$$
b_{0}(v, w)=a\left(\Pi_{C} v, \Pi_{C} w\right) \quad \forall v, w \in \mathscr{S}_{0}^{\prime}
$$

and

$$
b_{j}(v, w)=a(v, w) \quad \forall v, w \in \mathscr{S}_{j}^{\prime}, \quad j=1, \ldots, J
$$

where $\Pi_{C}$ be the interpolation operator which interpolates continuous functions into functions in $\mathscr{S}$.

We note that if $v_{0} \in \mathscr{S}_{0}$ and $v_{0}^{\prime} \in \mathscr{S}_{0}^{\prime}$ then

$$
v_{0}^{\prime}=\Pi_{F} v_{0} \Longleftrightarrow v_{0}=\Pi_{C} v_{0}^{\prime} .
$$

This subspace decomposition completely defines the additive Schwarz operator $P$ introduced in Section 3.

\subsection{Matrix form of additive Schwarz algorithm}

In this subsection we give the matrix formulation of the algorithm described in Section 3.

Coarse space correction: Let $\left\{\varphi_{k}: k \in \mathscr{N}_{C}\right\}$ be the set of nodal basis functions for $\mathscr{S}_{0}$ consisting of bilinear functions on the coarse grid. Then a basis for $\mathscr{S}$ is $\left\{\tilde{\varphi}_{k}: k \in \mathscr{N}_{C}\right\}$, where $\tilde{\varphi}_{k}=\Pi_{F} \varphi_{k}$. Recall the basis $\left\{\varphi_{l}: l \in \mathscr{N}\right\}$ for $\mathscr{S}$ (see Subsection 4.1). Each $\tilde{\varphi}_{k}$ can be represented as

$$
\tilde{\varphi}_{k}=\sum_{l \in \mathscr{N}} \varepsilon_{k, l} \varphi_{l}
$$

where $\varepsilon_{k, l}=\varphi_{k}\left(\mathbf{x}_{l}\right)$. Let

$$
\mathbf{R}_{0}=\left[\varepsilon_{k, l}\right]_{k \in \mathscr{N}_{C}, l \in \mathscr{N}} .
$$

Then $\mathbf{R}_{0}$ is the matrix that transforms the basis of $\mathscr{S}$ into the basis of $\mathscr{S}_{0}$.

Equation (3.4) for $j=0$ reads as: Find $u_{0}=\sum_{k \in \mathscr{N}_{C}} \alpha_{k} \tilde{\varphi}_{k} \in \mathscr{S}_{0}^{\prime}$ such that

$$
\sum_{k \in \mathscr{N}_{C}} \alpha_{k} b_{0}\left(\tilde{\varphi}_{k}, \tilde{\varphi}_{j}\right)=\left\langle r, \tilde{\varphi}_{j}\right\rangle, \quad j \in \mathscr{N}_{C}
$$

which is equivalent to, noting (4.2) and (4.4),

$$
\sum_{k \in \mathscr{N}_{C}} \alpha_{k} a\left(\varphi_{k}, \varphi_{j}\right)=\sum_{l \in \mathscr{N}} \varepsilon_{j, l}\left\langle r, \varphi_{l}\right\rangle, \quad j \in \mathscr{N}_{C} .
$$

For any $v=\sum_{k \in \mathscr{N}} v_{k} \varphi_{k} \in \mathscr{S}$, let $\mathbf{v}=\left(v_{k}\right)_{k \in \mathscr{N}}$. Then 
where $\mathbf{M}$ is the mass matrix defined as $\mathbf{M}=\left[\left(\varphi_{k}, \varphi_{l}\right)\right]_{k, l \in \mathscr{N}}$. Denoting by $\mathbf{A}_{0}$ the stiffness matrix $\left[a\left(\varphi_{k}, \varphi_{l}\right)\right]_{k, l \in \mathscr{N}_{C}}$ computed on the coarse grid (with bilinear functions) and by $\alpha$ the vector $\left(\alpha_{k}\right)_{k \in \mathscr{N}_{C}}$, we can rewrite (4.5) as

$$
\mathbf{A}_{0} \alpha=\mathbf{R}_{0} \mathbf{M r}
$$

implying

$$
\alpha=\mathbf{A}_{0}^{-1} \mathbf{R}_{0} \mathbf{M r}
$$

so that

$$
\mathbf{u}_{0}=\mathbf{R}_{0}^{t} \alpha=\mathbf{R}_{0}^{t} \mathbf{A}_{0}^{-1} \mathbf{R}_{0} \mathbf{M r}
$$

Corrections on $\mathscr{S}_{i}^{\prime}, \quad i=1, \ldots, J$ : A basis for $\mathscr{S}_{i}^{\prime}$ with $i=1, \ldots, J$ is more straightforward: it is extracted from $\left\{\varphi_{l}: l \in \mathscr{N}\right\}$ by a Boolean matrix $\mathbf{R}_{i}$. Using the same argument as above we obtain

$$
\mathbf{u}_{i}=\mathbf{R}_{i}^{t} \mathbf{A}_{i}^{-1} \mathbf{M}_{i} \mathbf{R}_{i} \mathbf{r}, \quad i=1, \ldots, J
$$

where

$$
\mathbf{A}_{i}=\mathbf{R}_{i} \mathbf{A} \mathbf{R}_{i}^{t}, \quad \mathbf{M}_{i}=\mathbf{R}_{i} \mathbf{M} \mathbf{R}_{i}^{t}
$$

By noting that $\mathbf{M}_{i} \mathbf{R}_{i}=\mathbf{R}_{i} \mathbf{M}$ we deduce

$$
\mathbf{u}_{i}=\mathbf{R}_{i}^{t} \mathbf{A}_{i}^{-1} \mathbf{R}_{i} \mathbf{M r}, \quad i=1, \ldots, J .
$$

Preconditioned residual: The preconditioned residual $u=B^{-1} r$ is computed by

$$
\mathbf{u}=\sum_{i=0}^{J} \mathbf{R}_{i}^{t} \mathbf{A}_{i}^{-1} \mathbf{R}_{i} \mathbf{M r}
$$

\subsection{The main result}

Theorem 4.1. The condition number of the additive Schwarz operator $P$ is bounded as

$$
\varkappa(P) \preceq 1+\log ^{2}(H / \delta) .
$$

Proof. The above estimate is a result of Lemma 3.1 and Lemmas 5.3 and 5.4

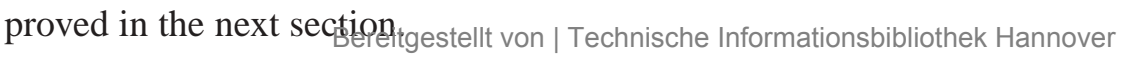




\section{TECHNICAL LEMMAS}

For the analysis, if $R \subset \mathbb{R}^{n}, n=1,2$, is a Lipschitz domain, we also define the following norms:

$$
\|v\|_{H^{1 / 2}(R)}^{2}:=\frac{1}{\operatorname{diam}(R)}\|v\|_{L_{2}(R)}^{2}+|v|_{H^{1 / 2}(R)}^{2}
$$

and

$$
\|v\|_{\widetilde{H}^{1 / 2}(R)}^{2}:=|v|_{H^{1 / 2}(R)}^{2}+\int_{R} \frac{v^{2}(\mathbf{x})}{\operatorname{dist}(\mathbf{x}, \partial R)} \mathrm{d} \mathbf{x}
$$

where

$$
|v|_{H^{1 / 2}(R)}^{2}:=\int_{R} \int_{R} \frac{|v(\mathbf{x})-v(\mathbf{y})|^{2}}{|\mathbf{x}-\mathbf{y}|^{n+1}} \mathrm{~d} \mathbf{x} \mathrm{d} \mathbf{y} .
$$

It was proved in Lemma 2 in [2] that if $R=[0, H] \times[0, H]$, then

$$
\|v\|_{\widetilde{H}^{1 / 2}(R)} \simeq\|v\|_{\widetilde{H}^{1 / 2}(R)} \quad \forall v \in \widetilde{H}^{1 / 2}(R)
$$

where the constants are independent of $H$. In the same manner one can prove that if $R=[0, H]$, then

$$
\|v\|_{H^{1 / 2}(R)} \simeq\|v\|_{H^{1 / 2}(R)} \quad \forall v \in H^{1 / 2}(R) .
$$

For the semi-norm $|\cdot|_{H^{1 / 2}(R)}$ with $R=I \times J$, where $I$ and $J$ are intervals, it is useful to use the following equivalent form, which was proved in Lemma 5.3 in [11] (see also Exercise 5.1 following that lemma)

$$
|v|_{H^{1 / 2}(R)}^{2} \simeq \int_{I} \int_{I} \frac{\left\|v(x, \cdot)-v\left(x^{\prime}, \cdot\right)\right\|_{L_{2}(J)}^{2}}{\left|x-x^{\prime}\right|^{2}} \mathrm{~d} x \mathrm{~d} x^{\prime}+\int_{J} \int_{J} \frac{\left\|v(\cdot, y)-v\left(\cdot, y^{\prime}\right)\right\|_{L_{2}(I)}^{2}}{\left|y-y^{\prime}\right|^{2}} \mathrm{~d} \mathrm{~d} y^{\prime}
$$

where the constants in the equivalence are independent of the sizes of $I, J$, and $R$.

The following results were proved in Lemma 3.5 in [6]; however, for the convenience of the reader we present the detailed proof to justify that the results still hold with the norm defined in (5.1).

Lemma 5.1. Let $0<\delta<\beta$.

1. If $v \in H^{1 / 2}(0, \beta)$, then

$$
\int_{\delta}^{\beta} \frac{|v(x)|^{2}}{x} \mathrm{~d} x \preceq\left(1+\log ^{2} \frac{\beta}{\delta}\right)\|v\|_{H^{1 / 2}(0, \beta)}^{2} .
$$

2. If $v \in H^{1 / 2}\left((0, \beta)^{2}\right)$, then

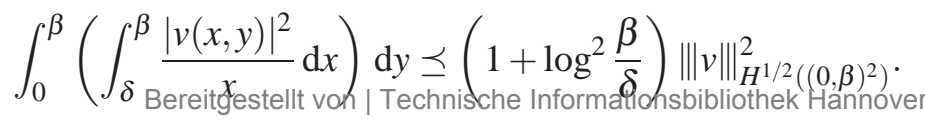


Proof. Let $\mathscr{S}_{\delta}$ be the space of continuous piecewise-linear functions on a uniform mesh of size $\delta$ on $(0, \beta)$. We first prove (5.7) for $v_{\delta} \in \mathscr{S}_{\delta}$. By noting that (see Lemma 3.3 in [6])

$$
\left\|v_{\delta}\right\|_{L_{\infty}(0, \beta)}^{2} \preceq\left(1+\log \frac{\beta}{\delta}\right)\left\|v_{\delta}\right\|_{H^{1 / 2}(0, \beta)}^{2}
$$

we have

$$
\int_{\delta}^{\beta} \frac{\left|v_{\delta}(x)\right|^{2}}{x} \mathrm{~d} x \leqslant\left\|v_{\delta}\right\|_{L_{\infty}(0, \beta)}^{2} \int_{\delta}^{\beta} \frac{\mathrm{d} x}{x} \preceq\left(1+\log ^{2} \frac{\beta}{\delta}\right)\left\|v_{\delta}\right\|_{H^{1 / 2}(0, \beta)}^{2} .
$$

Now consider $v \in H^{1 / 2}(0, \beta)$. Let $v_{\delta} \in \mathscr{S}_{\delta}$ be the $L_{2}$ projection of $v$ onto $\mathscr{S}_{\delta}$. Then

$$
\left\|v-v_{\delta}\right\|_{L_{2}(0, \beta)}^{2} \preceq \delta|v|_{H^{1 / 2}(0, \beta)}^{2} .
$$

Moreover, as was proved in [4] (see also [5]),

$$
\left\|v_{\delta}\right\|_{H^{1 / 2}(0, \beta)} \preceq\|v\|_{H^{1 / 2}(0, \beta)}
$$

which, together with (5.5), yields

$$
\left\|v_{\delta}\right\|_{H^{1 / 2}(0, \beta)} \preceq\|v\|_{H^{1 / 2}(0, \beta)} .
$$

Therefore,

$$
\begin{aligned}
& \int_{\delta}^{\beta} \frac{|v(x)|^{2}}{x} \mathrm{~d} x \preceq \int_{\delta}^{\beta} \frac{\left|v(x)-v_{\delta}(x)\right|^{2}}{x} \mathrm{~d} x+\int_{\delta}^{\beta} \frac{\left|v_{\delta}(x)\right|^{2}}{x} \mathrm{~d} x \\
& \preceq \frac{1}{\delta}\left\|v-v_{\delta}\right\|_{L_{2}(0, \beta)}^{2}+\int_{\delta}^{\beta} \frac{\left|v_{\delta}(x)\right|^{2}}{x} \mathrm{~d} x \\
& \preceq|v|_{H^{1 / 2}(0, \beta)}^{2}+\left(1+\log ^{2} \frac{\beta}{\delta}\right)\left\|v_{\delta}\right\|_{H^{1 / 2}(0, \beta)}^{2}
\end{aligned}
$$

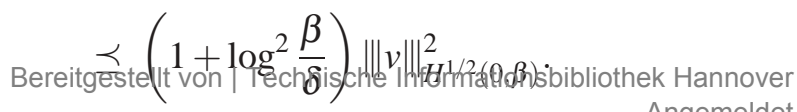


Inequality (5.8) is obtained by using (5.7) and (5.6)

$$
\begin{aligned}
\int_{0}^{\beta}\left(\int_{\delta}^{\beta} \frac{|v(x, y)|^{2}}{x} \mathrm{~d} x\right) \mathrm{d} y \preceq & \left(1+\log ^{2} \frac{\beta}{\delta}\right) \int_{0}^{\beta}\|v(\cdot, y)\|_{H^{1 / 2}(0, \beta)}^{2} \mathrm{~d} y \\
\preceq & \left(1+\log ^{2} \frac{\beta}{\delta}\right) \int_{0}^{\beta}\left[\frac{1}{\beta}\|v(\cdot, y)\|_{L_{2}(0, \beta)}^{2}\right. \\
& \left.+\int_{0}^{\beta} \int_{0}^{\beta} \frac{\left|v(x, y)-v\left(x^{\prime}, y\right)\right|^{2}}{\left|x-x^{\prime}\right|^{2}} \mathrm{~d} x \mathrm{~d} x^{\prime}\right] \mathrm{d} y \\
\preceq & \left(1+\log ^{2} \frac{\beta}{\delta}\right)\left[\frac{1}{\beta}\|v\|_{L_{2}\left((0, \beta)^{2}\right)}^{2}\right. \\
& \left.+\int_{0}^{\beta} \int_{0}^{\beta} \frac{\left\|v(x, \cdot)-v\left(x^{\prime}, \cdot\right)\right\|_{L_{2}(0, \beta)}^{2}}{\left|x-x^{\prime}\right|^{2}} \mathrm{~d} x \mathrm{~d} x^{\prime}\right] \\
\preceq & \left(1+\log ^{2} \frac{\beta}{\delta}\right)\|v\|_{H^{1 / 2}\left((0, \beta)^{2}\right)}^{2}
\end{aligned}
$$

finishing the proof of the lemma.

The above results will be used to prove the following lemma, which is crucial to obtain the estimate for the minimum eigenvalue of $P$ (see Lemma 3.1). Let $R$ be the union of overlapping rectangular subdomains $R, l=1, \ldots, J$, of diameters $\beta_{l}$. Assume that the size of the overlap is $\delta$. Let $\left\{\vartheta_{l}: l=1, \ldots, J\right\}$ be a partition of unity on $R$ defined by piecewise bilinear functions such that supp $\vartheta=\bar{R}_{l}$.

Lemma 5.2. If $\delta \leqslant \min _{l} \beta_{l} / 2$, then for any $w \in \widetilde{H}^{1 / 2}(R)$ there holds

$$
\sum_{l=1}^{J}\left\|\vartheta_{l} w\right\|_{\widetilde{H}^{1 / 2}\left(R_{l}\right)}^{2} \preceq \sum_{l=1}^{J}\left(1+\log \frac{\beta_{l}}{\delta}\right)^{2}\|w\|_{H^{1 / 2}\left(R_{l}\right)}^{2}+\int_{R} \frac{|w(\mathbf{x})|^{2}}{\operatorname{dist}(\mathbf{x}, \partial R)} \mathrm{d} \mathbf{x} .
$$

Proof. Without loss of generality, we can assume that $R_{l}=I \times I$, where $I=\left(0, \beta_{l}\right)$, so that $\vartheta_{l}$ can be defined as

$$
\vartheta_{l}(x, y)=\eta_{l}(x) \eta_{l}(y), \quad \eta_{l}(t)= \begin{cases}t / \delta, & 0 \leqslant t \leqslant \delta \\ 1, & \delta<t<\beta_{l}-\delta \\ \left(\beta_{l}-t\right) / \delta, & \beta_{l}-\delta \leqslant t \leqslant \beta_{l} .\end{cases}
$$

In view of (5.2) and (5.6) in order to estimate $\left\|\vartheta_{l} w\right\|_{\widetilde{H}^{1 / 2}\left(R_{l}\right)}^{2}$ we will estimate

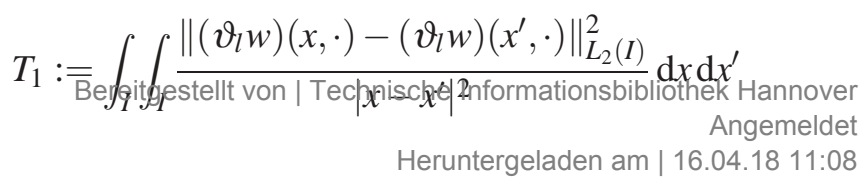


and

$$
T_{2}:=\int_{R_{l}} \frac{\left|\left(\vartheta_{l} w\right)(\mathbf{x})\right|^{2}}{\operatorname{dist}\left(\mathbf{x}, \partial R_{l}\right)} \mathrm{d} \mathbf{x}
$$

Splitting the integral over $I=\left(0, \beta_{l}\right)$ into three integrals over $I_{1}:=[0, \delta], I_{2}:=$ $\left[\delta, \beta_{l}-\delta\right]$, and $I_{3}:=\left[\beta_{l}-\delta, \beta_{l}\right]$, and denoting, for any $v$,

$$
A_{i j}(v):=\iint_{I_{i} \times I_{j}} \frac{\left\|v(x, \cdot)-v\left(x^{\prime}, \cdot\right)\right\|_{L_{2}(I)}^{2}}{\left|x-x^{\prime}\right|^{2}} \mathrm{~d} x \mathrm{~d} x^{\prime}
$$

we observe that, by symmetry, in order to estimate $T_{1}$ it suffices to consider $A_{11}\left(\vartheta_{l} w\right)$, $A_{12}\left(\vartheta_{l} w\right), A_{13}\left(\vartheta_{l} w\right), A_{23}\left(\vartheta_{l} w\right)$, and $A_{33}\left(\vartheta_{l} w\right)$. There is no need to consider $A_{22}\left(\vartheta_{l} w\right)$ because $A_{22}\left(\vartheta_{l} w\right)=A_{22}(w)$. The symmetric shape of $\vartheta_{l}$ implies the similarity of $A_{11}\left(\vartheta_{l} w\right)$ and $A_{33}\left(\vartheta_{l} w\right)$, and of $A_{12}\left(\vartheta_{l} w\right)$ and $A_{23}\left(\vartheta_{l} w\right)$. There remains three terms to be considered: $A_{11}\left(\vartheta_{l} w\right), A_{12}\left(\vartheta_{l} w\right)$, and $A_{13}\left(\vartheta_{l} w\right)$.

Firstly, for $A_{11}$ we have

$$
\begin{aligned}
A_{11}\left(\vartheta_{l} w\right) \leqslant & 2 \iint_{I_{1} \times I_{1}} \frac{\left\|\vartheta_{l}(x, \cdot)-\vartheta_{l}\left(x^{\prime}, \cdot\right)\right\|_{L_{2}(I)}^{2}}{\left|x-x^{\prime}\right|^{2}}\|w(x, \cdot)\|_{L_{2}(I)}^{2} \mathrm{~d} x \mathrm{~d} x^{\prime} \\
& +2 \iint_{I_{1} \times I_{1}} \frac{\left\|w(x, \cdot)-w\left(x^{\prime}, \cdot\right)\right\|_{L_{2}(I)}^{2}}{\left|x-x^{\prime}\right|^{2}}\left\|\vartheta_{l}(x, \cdot)\right\|_{L_{2}(I)}^{2} \mathrm{~d} x \mathrm{~d} x^{\prime} \\
& \leqslant 2 \iint_{I_{1} \times I_{1}} \frac{\sum_{i=1}^{3}\left\|\frac{x}{\delta} \eta(\cdot)-\frac{x^{\prime}}{\delta} \eta(\cdot)\right\|_{L_{2}\left(I_{i}\right)}^{2}}{\left|x-x^{\prime}\right|^{2}}\|w(x, \cdot)\|_{L_{2}(I)}^{2} \mathrm{~d} x \mathrm{~d} x^{\prime}+2 \beta_{l} A_{11}(w) \\
& \preceq \frac{1}{\delta}\|w\|_{L_{2}\left(I_{1} \times I\right)}^{2}+A_{11}(w) .
\end{aligned}
$$

It was proved in Lemma 3.4 in [6] that

$$
\frac{1}{\delta}\|w\|_{L_{2}\left(I_{1} \times I\right)}^{2} \leqslant c\left(1+\log \frac{\beta_{l}}{\delta}\right)\|w\|_{H^{1 / 2}\left(R_{l}\right)}^{2}
$$

where $c$ is independent of $w, \beta_{l}$, and $\delta$. Thus $A_{11}\left(\vartheta_{l} w\right)$ is bounded by the right-hand side of (5.9).

For the term $A_{12}\left(\vartheta_{l} w\right)$ we have

$$
\begin{aligned}
& A_{12}\left(\vartheta_{l} w\right)=\frac{1}{\delta^{2}} \int_{0}^{\delta} \int_{\delta}^{\beta_{l}-\delta} \frac{\left\|x w(x, \cdot)-\delta w\left(x^{\prime}, \cdot\right)\right\|_{L_{2}(I)}^{2}}{\left|x-x^{\prime}\right|^{2}} \mathrm{~d} x^{\prime} \mathrm{d} x \\
& \leqslant \frac{2}{\delta^{2}} \int_{0}^{\delta}\left(\int_{\delta}^{\beta_{l}-\delta} \frac{\mathrm{d} x^{\prime}}{\left|x-x^{\prime}\right|^{2}}\right)|x-\delta|^{2}\|w(x, \cdot)\|_{L_{2}(I)}^{2} \mathrm{~d} x+2 A_{12}(w)
\end{aligned}
$$

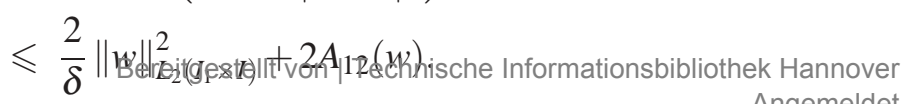


Using again (5.11) we deduce that $A_{12}\left(\vartheta_{l} w\right)$ is bounded by the right-hand side of (5.9).

Finally, for $A_{13}\left(\vartheta_{l} w\right)$ since the assumption $\delta \leqslant \beta_{l} / 2$ implies that $\beta_{l} / 2-x \geqslant 0$ for $x \in I_{1}$ and $x^{\prime}-\beta_{l} / 2 \geqslant 0$ for $x^{\prime} \in I_{3}$, we find

$$
\begin{aligned}
A_{13}\left(\vartheta_{l} w\right)= & \frac{1}{\delta^{2}} \int_{0}^{\delta} \int_{\beta_{l}-\delta}^{\beta_{l}} \frac{\left\|x w(x, \cdot)-\left(\beta_{l}-x^{\prime}\right) w\left(x^{\prime}, \cdot\right)\right\|_{L_{2}(I)}^{2}}{\left|x-x^{\prime}\right|^{2}} \mathrm{~d} x^{\prime} \mathrm{d} x \\
\leqslant & \frac{2}{\delta^{2}} \int_{0}^{\delta} \int_{\beta_{l}-\delta}^{\beta_{l}} \frac{\left|x+x^{\prime}-\beta_{l}\right|^{2}}{\left|x-x^{\prime}\right|^{2}}\|w(x, \cdot)\|_{L_{2}(I)}^{2} \mathrm{~d} x^{\prime} \mathrm{d} x \\
& +\frac{2}{\delta^{2}} \int_{0}^{\delta} \int_{\beta_{l}-\delta}^{\beta_{l}}\left|\beta_{l}-x^{\prime}\right|^{2} \frac{\left\|w(x, \cdot)-w\left(x^{\prime}, \cdot\right)\right\|_{L_{2}(I)}^{2}}{\left|x-x^{\prime}\right|^{2}} \mathrm{~d} x^{\prime} \mathrm{d} x \\
\leqslant & \frac{2}{\delta^{2}} \int_{0}^{\delta} \int_{\beta_{l}-\delta}^{\beta_{l}} \frac{\left|\left(x^{\prime}-\frac{\beta_{l}}{2}\right)-\left(\frac{\beta_{l}}{2}-x\right)\right|^{2}}{\left|\left(x^{\prime}-\frac{\beta_{l}}{2}\right)+\left(\frac{\beta_{l}}{2}-x\right)\right|^{2}}\|w(x, \cdot)\|_{L_{2}(I)}^{2} \mathrm{~d} x^{\prime} \mathrm{d} x+2 A_{13}(w) \\
\leqslant & \frac{2}{\delta}\|w\|_{L_{2}\left(I_{1} \times I\right)}^{2}+2 A_{13}(w) .
\end{aligned}
$$

Inequality (5.11) yields the estimate for $A_{13}\left(\vartheta_{l} w\right)$, which implies that $T_{1}$ is bounded by the right-hand side of (5.9).

To estimate $T_{2}$ we first assume, without loss of generality, that $\operatorname{dist}\left(\mathbf{x}, \partial R_{l}\right)=x$, where $\mathbf{x}=(x, y)$. Then

$$
\begin{aligned}
T_{2} & \leqslant \int_{0}^{\beta_{l}}\left[\int_{0}^{\delta} \frac{x^{2}}{\delta^{2}} \frac{|w(x, y)|^{2}}{x} \mathrm{~d} x+\int_{\delta}^{\beta_{l}} \frac{|w(x, y)|^{2}}{x} \mathrm{~d} x\right] \mathrm{d} y \\
& \leqslant \frac{1}{\delta}\|w\|_{L_{2}\left(I_{1} \times I\right)}^{2}+\int_{0}^{\beta_{l}} \int_{\delta}^{\beta_{l}} \frac{|w(x, y)|^{2}}{x} \mathrm{~d} x \mathrm{~d} y .
\end{aligned}
$$

The desired estimate for $T_{2}$ now follows from (5.11) and (5.8), finishing the proof.

Lemma 5.3. For any $u \in \mathscr{S}$ there exist $u_{i} \in \mathscr{S}_{i}^{\prime}$ satisfying $u=\sum_{i=0}^{J} u_{i}$ such that

$$
\sum_{i=0}^{J} b_{i}\left(u_{i}, u_{i}\right) \preceq\left(1+\log ^{2} \frac{H}{\delta}\right) a(u, u),
$$

where the constant is independent of $u, H, h$, and $\delta$.

Proof. To define a decomposition for $u \in \mathscr{S}$ we need a projection and a partition of unity. Since the operator $-\Delta$ with domain of definition $\widetilde{H}^{1}(\Gamma)=H_{0}^{1}(\Gamma)$ is positive definite and self-adjoint, we can define $\Lambda=\sqrt{-\Delta}$ which in turn is self-adjoint as an operator from $\widetilde{H}^{1 / 2}(\Gamma)$ to $H^{-1 / 2}(\Gamma)$. Moreover,

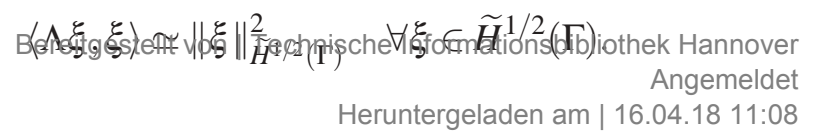


Let $P_{H}: \widetilde{H}^{1 / 2}(\Gamma) \longrightarrow \mathscr{S}_{0}$ be the projection defined by the inner product $\langle\Lambda \cdot, \cdot\rangle$, i.e.,

$$
\left\langle\Lambda P_{H} v, w\right\rangle=\langle\Lambda v, w\rangle \quad \forall v \in \widetilde{H}^{1 / 2}(\Gamma), w \in \mathscr{S}_{0} .
$$

Using standard arguments one can prove that for any $v \in \widetilde{H}^{1 / 2}(\Gamma)$ there hold

$$
\left\|P_{H} v\right\|_{\widetilde{H}^{1 / 2}(\Gamma)} \preceq\|v\|_{\widetilde{H}^{1 / 2}(\Gamma)}, \quad\left\|P_{H} v-v\right\|_{L_{2}(\Gamma)} \preceq H^{1 / 2}\|v\|_{\widetilde{H}^{1 / 2}(\Gamma)} .
$$

We next define a partition of unity having the properties of the partition in Lemma 5.2. For $j=1, \ldots, J$ we define

$$
R_{j}=\Gamma_{j} \cup\left\{\mathbf{x} \in \Gamma: \operatorname{dist}\left(\mathbf{x}, \partial \Gamma_{j}\right) \leqslant \delta\right\}
$$

which are quadrilateral domains (see Fig. 1). It is crucial to define $R_{j}$ as a quadrilateral domain so that Lemma 5.2 can be applied. The cutoff function $\vartheta_{j}$ is defined as in the proof of Lemma 5.2 so that supp $\vartheta_{j}=\bar{R}_{j}$.

We are now ready to define a decomposition of $u \in \mathscr{S}$ such that (5.12) holds. For any $u \in \mathscr{S}$ let $u_{0}=\Pi_{F} P_{H} u \in \mathscr{S}_{0}^{\prime}$ and $u_{j}=\Pi_{F}\left(\vartheta_{j} w\right) \in \mathscr{S}_{j}^{\prime}, j=1, \ldots, J$, where $w=u-u_{0}$. The fact that supp $u_{j} \subset \bar{\Gamma}_{j}^{\prime}$ so that $u_{j} \in \mathscr{S}_{j}^{\prime}$ for $j=1, \ldots, J$ is clear from the definitions of $R_{j}$ and $\Gamma_{j}^{\prime}$. It is also clear that $u=\sum_{i=0}^{J} u_{i}$. By using (4.4), (5.13) and Lemma 7 in [2], we obtain the following estimates

$$
\begin{aligned}
\left\|\Pi_{C} u_{0}\right\|_{\widetilde{H}^{1 / 2}(\Gamma)} & =\left\|P_{H} u\right\|_{\widetilde{H}^{1 / 2}(\Gamma)} \\
& \preceq\|u\|_{\widetilde{H}^{1 / 2}(\Gamma)} \\
\left\|u_{i}\right\|_{\widetilde{H}^{1 / 2}(\Gamma)} & \preceq\left\|\vartheta_{i} w\right\|_{\widetilde{H}^{1 / 2}(\Gamma)} \\
\|w\|_{\widetilde{H}^{1 / 2}(\Gamma)} & =\left\|\Pi_{F}\left(u-P_{H} u\right)\right\|_{\widetilde{H}^{1 / 2}(\Gamma)} \\
& \preceq\left\|u-P_{H} u\right\|_{\widetilde{H}^{1 / 2}(\Gamma)} \\
& \preceq\|u\|_{\widetilde{H}^{1 / 2}(\Gamma)}
\end{aligned}
$$

and

$$
\begin{aligned}
\|w\|_{L_{2}(\Gamma)} & =\left\|\Pi_{F}\left(u-P_{H} u\right)\right\|_{L_{2}(\Gamma)} \\
& \preceq\left\|u-P_{H} u\right\|_{L_{2}(\Gamma)} \\
& \preceq H^{1 / 2}\|u\|_{\widetilde{H}^{1 / 2}(\Gamma)} .
\end{aligned}
$$

Moreover, by Lemma 6 in [2] there holds 
Hence by using successively (2.4), (5.14), (5.15), (5.4), (5.18), (5.9), (4.1), (5.16), and (5.17) we obtain

$$
\begin{aligned}
\sum_{i=0}^{J} b_{i}\left(u_{i}, u_{i}\right) & \simeq\left\|\Pi_{C} u_{0}\right\|_{\widetilde{H}^{1 / 2}(\Gamma)}^{2}+\sum_{i=1}^{J}\left\|u_{i}\right\|_{\widetilde{H}^{1 / 2}(\Gamma)}^{2} \\
& \preceq\|u\|_{\widetilde{H}^{1 / 2}(\Gamma)}^{2}+\sum_{i=1}^{J}\left\|\vartheta_{i} w\right\|_{\widetilde{H}^{1 / 2}(\Gamma)}^{2} \\
& \simeq\|u\|_{\widetilde{H}^{1 / 2}(\Gamma)}^{2}+\sum_{i=1}^{J}\left\|\vartheta_{i} w\right\|_{\widetilde{H}^{1 / 2}(\Gamma)}^{2} \\
& \simeq\|u\|_{\widetilde{H}^{1 / 2}(\Gamma)}^{2}+\sum_{i=1}^{J}\left\|\vartheta_{i} w\right\|_{\widetilde{H}^{1 / 2}\left(R_{i}\right)}^{2} \\
& \preceq\|u\|_{\widetilde{H}^{1 / 2}(\Gamma)}^{2}+\left(1+\log ^{2} \frac{H}{\delta}\right) \sum_{i=1}^{J}\|w\|_{H^{1 / 2}\left(R_{i}\right)}^{2}+\int_{\Gamma} \frac{|w(x)|^{2}}{\operatorname{dist}(x, \partial \Gamma)} \mathrm{d} x \\
& \preceq\|u\|_{\widetilde{H}^{1 / 2}(\Gamma)}^{2}+\left(1+\log ^{2} \frac{H}{\delta}\right) \sum_{i=1}^{J}\|w\|_{H^{1 / 2}\left(R_{i}\right)}^{2}+\|w\|_{\widetilde{H}^{1 / 2}(\Gamma)}^{2} \\
& \preceq\|u\|_{\widetilde{H}^{1 / 2}(\Gamma)}^{2}+\left(1+\log ^{2} \frac{H}{\delta}\right) \sum_{i=1}^{J} \frac{1}{H}\|w\|_{L_{2}\left(R_{i}\right)}^{2} \\
& \preceq\|u\|_{\widetilde{H}^{1 / 2}(\Gamma)}^{2}+\left(1+\log ^{2} \frac{H}{\delta}\right) \frac{1}{H}\|w\|_{L_{2}(\Gamma)}^{2} \\
& \preceq\left(1+\log ^{2} \frac{H}{\delta}\right)\|u\|_{\widetilde{H}^{1 / 2}(\Gamma)}^{2} \\
& \preceq\left(1+\log ^{2} \frac{H}{\delta}\right) a(u, u)
\end{aligned}
$$

completing the proof.

Lemma 5.4. For any $u \in \mathscr{S}$ if $u=\sum_{i=0}^{J} u_{i}$ for some $u_{i} \in \mathscr{S}_{i}$, then

$$
a(u, u) \preceq \sum_{i=0}^{J} b_{i}\left(u_{i}, u_{i}\right)
$$

where the constant is independent of $u, h, \delta$, and $H$.

Proof. By construction there are at most $v$ subdomains $\bar{\Gamma}_{i}^{\prime}$ to which any $x \in \Gamma$

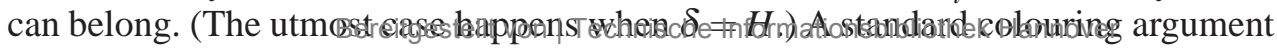


Table 1.

Condition numbers for Laplace equation, overlap size $\delta=h$.

\begin{tabular}{rlccc}
\hline DoF & $h$ & Unpreconditioned & $H=2 h$ & $H=1 / 2$ \\
\hline 9 & $1 / 4$ & 1.83 & 4.51 & 1.58 \\
49 & $1 / 8$ & 3.49 & 5.12 & 5.12 \\
225 & $1 / 16$ & 6.94 & 5.38 & 4.85 \\
961 & $1 / 32$ & 13.84 & 5.46 & 5.16 \\
3969 & $1 / 64$ & 27.77 & 5.47 & 6.02 \\
16129 & $1 / 128$ & 55.73 & 5.48 & 7.29 \\
65025 & $1 / 256$ & 111.68 & 5.47 & 8.93 \\
261121 & $1 / 512$ & 223.60 & 5.54 & 10.92 \\
\hline
\end{tabular}

yields, noting that $u_{0}=\Pi_{F} \Pi_{C} u_{0}$,

$$
\begin{aligned}
a(u, u) & \simeq\|u\|_{\widetilde{H}^{1 / 2}(\Gamma)}^{2} \\
& \preceq\left\|u_{0}\right\|_{\widetilde{H}^{1 / 2}(\Gamma)}^{2}+\left\|\sum_{i=1}^{J} u_{i}\right\|_{\widetilde{H}^{1 / 2}(\Gamma)}^{2} \\
& \preceq\left\|\Pi_{C} u_{0}\right\|_{\widetilde{H}^{1 / 2}(\Gamma)}^{2}+v \sum_{i=1}^{J}\left\|u_{i}\right\|_{\widetilde{H}^{1 / 2}(\Gamma)}^{2} \\
& \simeq \sum_{i=0}^{J} b_{i}\left(u_{i}, u_{i}\right)
\end{aligned}
$$

completing the proof.

\section{NUMERICAL EXPERIMENTS}

We solved (2.2) for both the Laplace and Lamé operators, i.e. with $D$ defined by (2.3), and with $\Gamma$ chosen to be $[-1,1]^{2}$. The right hand side $g$ of (2.2) is chosen to be $g(x) \equiv 1$ in the Laplace case, and $g(x)=(-y, x, 0)$ in the Lamé case.

The experiments are performed with numerous values of $H, h$, and $\delta$, but we only report a few cases here. All numbers seem to agree with our theoretical result. Tables 1 and 2 present the condition number for both cases, whereas Tables 3 and 4 present the corresponding number of iterations. We also plot in Fig. 2 the condition number versus $H / \delta$ for the Laplace case. A logarithmic growth can be observed. The authors are grateful to Dr. Matthias Maischak for carrying out the experiments.

Acknowledgments. Parts of the paper were written when the first author was visiting the University of Hannover (supported by the DFG Graduiertenkolleg GRK615). The paper was finished when the second author was visiting the Univer-

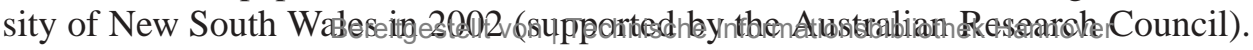


Table 2.

Condition number for Lamé equation.

\begin{tabular}{rlccccc}
\hline DoF & $h$ & Unpreconditioned & \multicolumn{2}{c}{$H=2 h$} & \multicolumn{2}{c}{$H=4 h$} \\
& & & $\delta=h$ & $\delta=2 h$ & $\delta=h$ & $\delta=2 h$ \\
\hline 147 & $1 / 8$ & 4.14 & 2.38 & 4.11 & & \\
675 & $1 / 16$ & 8.24 & 2.78 & 4.27 & 5.15 & 9.01 \\
2883 & $1 / 32$ & 16.50 & 3.88 & 4.31 & 5.43 & 9.01 \\
11907 & $1 / 64$ & 33.07 & 5.36 & 5.88 & 5.56 & 9.01 \\
\hline
\end{tabular}

Table 3.

Number of iterations for Laplace equation, overlap size $\delta=h$.

\begin{tabular}{rlccc}
\hline DoF & $h$ & Unpreconditioned & $H=2 h$ & $H=1 / 2$ \\
\hline 9 & $1 / 4$ & 4 & 4 & 4 \\
49 & $1 / 8$ & 11 & 15 & 15 \\
225 & $1 / 16$ & 16 & 20 & 17 \\
961 & $1 / 32$ & 23 & 21 & 18 \\
3969 & $1 / 64$ & 31 & 21 & 19 \\
16129 & $1 / 128$ & 44 & 21 & 20 \\
65025 & $1 / 256$ & 63 & 20 & 23 \\
261121 & $1 / 512$ & 89 & 20 & 24 \\
\hline
\end{tabular}

Table 4.

Number of iterations for Lamé equation.

\begin{tabular}{rlccccc}
\hline DoF & $h$ & Unpreconditioned & \multicolumn{2}{c}{$H=2 h$} & \multicolumn{2}{c}{$H=4 h$} \\
& & & $\delta=h$ & $\delta=2 h$ & $\delta=h$ & $\delta=2 h$ \\
\hline 147 & $1 / 8$ & 24 & 13 & 16 & & \\
675 & $1 / 16$ & 34 & 16 & 19 & 20 & 22 \\
2883 & $1 / 32$ & 48 & 19 & 21 & 22 & 25 \\
11907 & $1 / 64$ & 68 & 23 & 23 & 24 & 26 \\
\hline
\end{tabular}




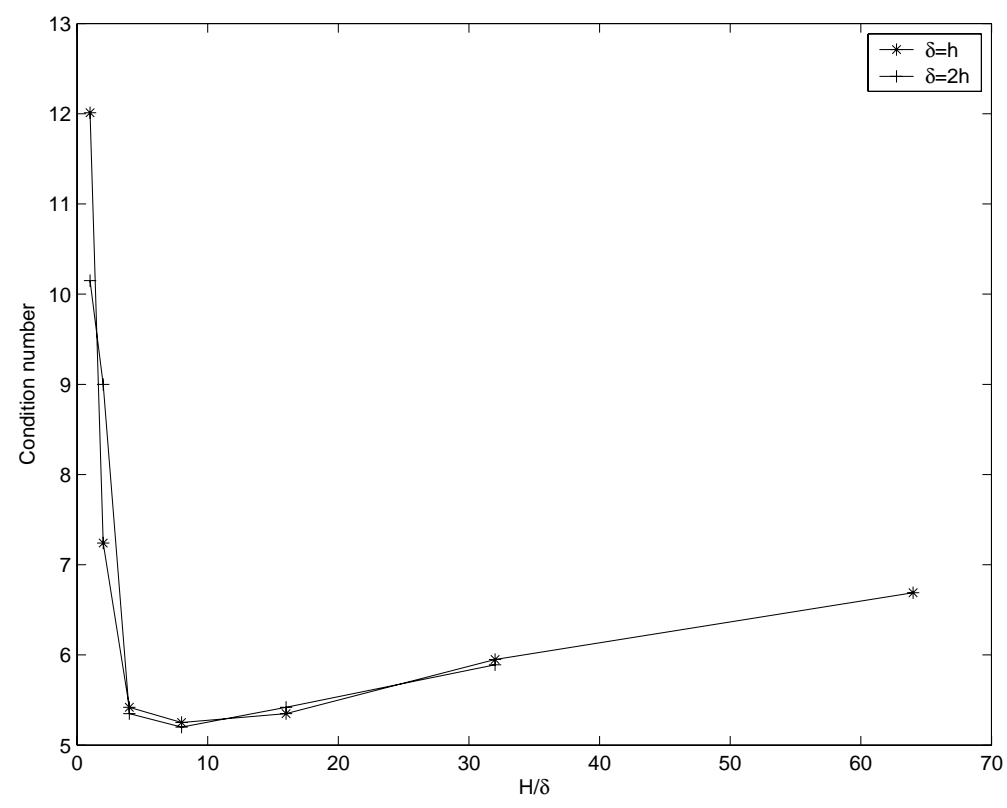

Figure 2. Condition number vs $H / \delta$ for $\mathrm{DoF}=16129$.

\section{REFERENCES}

1. M. Ainsworth and B. Guo, An additive Schwarz preconditioner for $p$-version boundary element approximation of the hypersingular operator in three dimensions. Numer. Math. (2000) 85, 343 366.

2. M. Ainsworth and B. Guo, Analysis of iterative sub-structuring techniques for boundary element approximation of the hypersingular operator in three dimensions. Appl. Anal. (2002) 81, $241-$ 280.

3. J. Bergh and J. Löfström, Interpolation Spaces: An Introduction. Springer-Verlag, Berlin, 1976.

4. J. Bramble, J. Pasciak, and O. Steinbach, On the stability of the $L^{2}$ projection in $H^{1}(\Omega)$. Math. Comp. (2001) 71, $147-156$.

5. C. Carstensen, Merging the Bramble-Pasciak-Steinbach and the Crouzeix-Thomée criterion for $H^{1}$-stability of the $L^{2}$-projection onto finite element spaces. Math. Comp. (2001) 71, $157-163$.

6. M. Dryja and O. B. Widlund, Domain decomposition algorithms with small overlap. SIAM J. Sci. Comput. (1994) 15, $604-620$.

7. H. Han, The boundary integro-differential equations of the three-dimensional Neumann problem in linear elasticity. Numer. Math. (1994) 68, 269-281.

8. N. Heuer and E.P. Stephan, Iterative substructuring for hypersingular integral equations in $\mathbb{R}^{3}$. SIAM J.Sci. Comput. (1999) 20, $739-749$.

9. N. Heuer and E. P. Stephan, An overlapping domain decomposition preconditioner for high order BEM with anisotropic elements. Advances in Comput. Math. (2003) 19, 211 - 230.

10. S. V. Nepomnyaschikh, Domain decomposition and Schwarz methods in a subspace for approxiBereitgestellt von |'Technische Informationsbibliothek Hannover 
mate solution of elliptic boundary value problems. PhD thesis, Computing Center of the Siberian Branch of the USSR Academy of Sciences, Novosibirsk, USSR, 1986.

11. J. Nečas, Les Méthodes Directes en Théorie des Equations Elliptiques. Masson et Cie, Paris, 1967.

12. B.F. Smith, An optimal domain decomposition preconditioner for the finite element solution of linear elasticity problems. SIAM J. Sci. Stat. Comput. (1992) 13, 364-378.

13. E. P. Stephan, A boundary integral equation method for three-dimensional crack problems in elasticity. Math. Meth. Appl. Sci. (1986) 8, 609-623.

14. E. P. Stephan, Boundary integral equations for screen problems in $\mathbb{R}^{3}$. Integral Eqns Operator Theory (1987) 10, 236-257.

15. T. Tran, Overlapping additive Schwarz preconditioners for boundary element methods. J. Integral Eqns Appl. (2000) 12, $177-207$.

16. T. Tran and E. P. Stephan, Additive Schwarz methods for the $h$ version boundary element method. Appl. Anal. (1996) 60, $63-84$.

17. T. Tran and E. P. Stephan, Additive Schwarz algorithms for the $p$ version of the Galerkin boundary element method. Numer. Math. (2000) 85, $433-468$.

18. O. Widlund, Optimal iterative refinement methods. In: Proc. of the Second International Symposium on Domain Decomposition Methods for Partial Differential Equations, (Eds. T. F. Chan, R. Glowinski, J. Périaux, and O. B. Widlund) Philadelphia, PA, SIAM, 1989, pp. 114 - 125, 